\title{
The value of the added diffusion-weighted images to multiparametric MRI in the early diagnosis of uterine cervix cancers and nodal assessment
}

\author{
Nasr Mohamed Osman and Mohamed Aboul-fotouh Mourad*i)
}

\begin{abstract}
Background: Cervical cancer still one of the most common causes of tumor-related death in developing countries presented in younger women. In this study, we aimed to evaluate the value of diffusion-weighted MRI in early diagnosis of malignant cervical lesions, to assess metastatic adenopathy, peritoneal dissemination, and possible tumor recurrence, and determine treatment response. This study included 60 patients with abnormal vaginal bleeding and suspected cervical lesion by US. A histopathological biopsy was done. Pelvic MR with DWI and dynamic contrast-enhanced MRI were done for all patients.

Results: According to the histopathological findings, we divided our studied 60 patents into two groups: group I, malignant lesions (46 lesions; 76.7\%), and group II, benign lesions (14 lesions; 23.3\%). Multiparametric MRI could detect all cervical lesions but with poor pathologic characterization, achieving $72.37 \%$ sensitivity, $37.50 \%$ specificity, 63.33\% accuracy, 76.19\% PPV, and 33.33\% NPV. When compared with DWI with ADC value measurements at high $b$ value $(b=800)$ to MRI exam, it showed a higher diagnostic accuracy with good lesion pathological characterization that achieved $95.65 \%$ sensitivity, $71.43 \%$ specificity, 90\% accuracy, 91.67 PPV, and 83.33\% NPV. The mean ADC value for malignant lesions was $0.86-1.1$, mean $=0.92 \pm 0.71 \times 10^{-3} \mathrm{~mm}^{2} / \mathrm{s}$, while the mean ADC value in the benign lesion group was $1.18 \pm 0.1 \times 10^{-3} \mathrm{~mm}^{2} / \mathrm{s}$.
\end{abstract}

Conclusion: Comparing DWI with ADC values measurements at high $b$ value to the multiparametric MRI examination of the female pelvis increases the sensitivity, specificity, and diagnostic accuracy of characterization and early diagnosis of cervical malignant focal lesions and reduces the need for intravenous contrast administration.

Keywords: Cancer cervix, DWI, Multiparametric MRI, Lymph node metastasis

\section{Background}

Cervical carcinoma is a common gynecological tumor still representing important cause of tumor-related death in younger females in developing countries [1]. The incidence rate of cervical cancer in Egypt is 6.6 cases/100, 000 populations, while prevalence of pre-invasive highgrade lesions represents $0.3 \%$ among the Egyptian

\footnotetext{
*Correspondence: m_aboul.fotouh@yahoo.com;

Mohamed.Mourad@mu.edu.eg

Diagnostic Radiology Department, Faculty of Medicine, Minia University, Minya, Egypt
}

\section{Springer Open}

females [2]. MRI could identify the anatomic origin, shape, and composition of uterine cervical masses, so a definitive diagnosis can be reached [3-5]. DW-MR imaging is a functional imaging technique whose contrast derives from the random motion of water molecules within tissues that allow its use in abdominal and pelvic applications [6]. DWI when combined with multiparametric MRI becomes a complementary diagnostic tool for the diagnosis of uterine lesions giving more information for the differentiation and extension of benign and malignant lesions [7, 8]. DWI is not only helpful in 
differentiating benign from malignant lesions but also it can be used to assess metastatic adenopathy, peritoneal dissemination, and possible tumor recurrence and determine treatment response [9].

This study aimed to evaluate the value of diffusionweighted MRI in early diagnosis of malignant cervical lesions.

\section{Methods}

This study included 60 patients with history of vaginal bleedings and suspicious cervical focal lesions by ultrasound examination. The patient's age ranged from 37 to 63 years with mean age \pm 44.6 years. Ethics committee approvals in addition to informed written consent were obtained from all included patients.

\section{Study population}

\section{Inclusion criteria}

Patients with clinical history of abnormal vaginal bleedings with suspicious transvaginal ultrasound findings of uterine cervix focal lesion were included. The patient did not receive any treatment prior to the examination.

\section{Exclusion criteria}

Patients unfit for MRI examination (e.g., who had cardiac pacemakers/prosthetic heart valves, cochlear implants, or other metallic implants) or having a history of claustrophobia or those who could not tolerate MRI exam were excluded.

\section{MRI examination}

MR imaging was performed on a 1.5- $\mathrm{T}$ scanner (Achieva, Philips Medical System) using pelvic phasedarray Torso coil with the patient in the supine position. Intravenous administration of an antispasmodic drug (10 mg of [Visceralgine; Organon, Livron, France]) was given immediately before MR imaging to reduce bowel peristalsis. All cases were asked to check their renal function (creatinine level) before the examination.

\section{MR imaging protocol inclusion}

The MR imaging protocol included the following:

- Non contrast MRI images, axial T1-weighted (TR/ TE, 500/10 ms), axial T2-weighted (TR/TE, 3300/ $100 \mathrm{~ms}$ ), slice thickness $6 \mathrm{~mm}$, gap $1 \mathrm{~mm}$, and FOV $32-42 \mathrm{~cm}$. Matrix $256 \times 256$. Coronal and sagittal T2-weighted, slice thickness $8-10 \mathrm{~mm}$, gap $1 \mathrm{~mm}$, and FOV 40-50 cm. Matrix $256 \times 256$.

- Gadopentetate dimeglumine contrast (Magnevist; Schering, Berlin, and Germany) was administered intravenously at weight-based dosing of $0.1 \mathrm{ml} / \mathrm{kg}$ body weight with a bolus injection rate of $2 \mathrm{ml} / \mathrm{s}$ by an automatic injector, followed by a $20 \mathrm{~mL}$ normal saline flushing the tube. Post-contrast T1 fat sat THRIVE (high-resolution isotropic volume examination) images were acquired instantly after administration of gadolinium. Images were acquired serially at $0,30,60,90$, and $120 \mathrm{~s}$.

- DW-MRI: using a spin echo single-shot sequence with free-breathing, with TR $2.8 \mathrm{~s}$, TE 72, matrix $512 \times 512$, slice-thickness $3 \mathrm{~mm}$ with an inter-slice gap of $1 \mathrm{~mm}$, and FOV of $350 \mathrm{~mm}$, and b-factor of 0,300 , and $800 \mathrm{~s} / \mathrm{mm}^{2}$ on an axial plane for each patient prior to contrast administration. The ADC maps were automatically generated for all DW images, and ADC values were measured at $b$ value $1000 \mathrm{~s} / \mathrm{mm}^{2}$. The mean ADC value was measured by placing ROI of average $1-2 \mathrm{~cm}$ in the solid part of the lesion and expressed in $10^{-3} \mathrm{~mm}^{2} / \mathrm{s}$.

\section{Histopathological correlation}

The histopathological results were used as a gold stander reference for all lesions and correlated to the imaging findings.

\section{Statistical analysis}

All statistical calculations were done using the SPSS test for calculation of mean, standard deviation, frequencies, and percentages. Sensitivity, specificity, accuracy, positive predictive value, and negative predictive value for MRI and DWI were calculated separately for each parameter.

\section{Results}

Two radiologists with 10 and 5 years' experience in gynecological MR imaging independently assessed cervical lesions of pelvic MRI examination with no available histopathological data. MRI imaging was evaluated for the following: the site, size, and extension of the cervical focal lesion, pattern of enhancement, infiltration of the related structures, and presence or absence of pelvic lymph nodes.

In the studied 60 suspicious uterine cervix focal lesions, the majority (21 patients) were complaining of abnormal vaginal bleeding, usually post-coital. Malodorous discharge and vaginal discomfort were reported in the remaining 9 patients. The patient's age ranged from 39 to 63 years with a mean age \pm 44.6 years.

\section{Histopathological results of studied 60 patients}

According to the histopathological findings, we divided our studied 60 patients into two groups: group I, malignant lesions group (46 cases; 76.7\%), and group II, benign lesions (14 cases; 23.3\%) (Table 1). 
Table 1 Histopathological results of the studied 60 lesions

\begin{tabular}{lll}
\hline Lesions & No. & Percent \% \\
\hline Group I: malignant lesions & $\mathbf{4 6}$ & 76.7 \\
Squamous cell carcinoma & $\mathbf{3 4}$ & 56.7 \\
Adenocarcinoma & $\mathbf{8}$ & 13.3 \\
Undifferentiated carcinoma & $\mathbf{4}$ & 6.7 \\
Group II: benign lesions & $\mathbf{1 4}$ & 23.3 \\
Cervical polyp (epithelial and endocervical) & $\mathbf{8}$ & 13.3 \\
Chronic cervicitis with nabothian cyst & $\mathbf{4}$ & 6.7 \\
Cervical leiomyomas & $\mathbf{2}$ & 3.3 \\
Total & 60 & 100 \\
\hline
\end{tabular}

Multiparametric MRI results in all studied uterine cervix focal lesions (number $=60$ cases)

Fifty-four lesions out of the studied 60 focal lesions of the uterine cervix found showed iso- to hypointense signals on T1WI and exhibited moderate hyper-intense signal on T2WI regardless of benign or malignant etiology. In the remaining 6 lesions, 4 of them were diagnosed as chronic cervicitis with nabothian cyst that showed iso- to low signal on T1WI and mixed intermediate-high signals in T2WI, and the remaining 2 cervical lesions were diagnosed to be degenerated leiomyoma which exhibits low signals on T1WI and mixed signals on T2WI (Table 2).

DW-MRI with ADC value measurement results of group I: malignant uterine cervix focal lesions (number $=46$ )

All studied malignant uterine cervix focal lesions (46 lesions) showed restricted diffusion on DW-MRI (diffusion positive) being of hyper-intense signals at DWI with persistent high signals at high $b$ value $(b=800)$ and corresponding low signal intensity at ADC map images. The ADC value of studied malignant endometrial lesions showed relatively low value averages of $0.86-1.15 \times 10^{-3}$ $\mathrm{mm}^{2} / \mathrm{s}$ with mean $\mathrm{ADC}$ value of about $0.92 \pm 0.71 \times$ $10^{-3} \mathrm{~mm}^{2} / \mathrm{s}$ (Table 3) (Figs. 1 and 2).

DW-MRI with ADC value measurement results in group II: benign uterine cervix focal lesions (number $=14$ )

Out of the studied 14 benign uterine lesions, 8 lesions which proved to be cervical polyps were diffusion negative in the form of low signals in DWI with high signals in low $b$ value that fade out in high $b$ value $(b=$ $800)$ with relatively high $\mathrm{ADC}$ value that ranged from 1.37 to $1.64 \times 10^{-3} \mathrm{~mm}^{2} / \mathrm{s}$ with a mean $\mathrm{ADC}$ value of about $1.4 \pm 0.31 \times 10^{-3} \mathrm{~mm}^{2} / \mathrm{s}$, while the 4 chronic cervicitis lesions with nabothian cyst showed mixed diffusion changes in the form of mild diffusion restriction with facilitated cystic areas, with $\mathrm{ADC}$ value ranging from 1.2 to $1.8 \times 10^{-3} \mathrm{~mm}^{2} / \mathrm{s}$ and with mean ADC value of about $1.5 \pm 0.31 \times 10^{-3} \mathrm{~mm}^{2} / \mathrm{s}$ and 2 uterine cervices degenerated fibroid that showed mixed diffusion changes evidenced as low signal with hyperintense foci in DWI and mixed low and high signals at ADC map, with ADC values $0.86 \times 10^{-3} \mathrm{~mm}^{2} / \mathrm{s}$. The $\mathrm{ADC}$ value of $5 / 7$ benign focal lesions showed relatively high values that ranged from 1.57 to $2.4 \times 10^{-3} \mathrm{~mm}^{2} / \mathrm{s}$ with a mean $\mathrm{ADC}$ value of about $1.7 \pm 0.31 \times 10^{-3} \mathrm{~mm}^{2} / \mathrm{s}$ with exception of 4 cervicitis with nabothian cyst and 2 degenerated fibroid that showed low ADC values which ranged from 0.86 to $1.2 \times 10^{-3} \mathrm{~mm}^{2} / \mathrm{s}$ with a mean $\mathrm{ADC}$ value of about $0.1 \pm$ 0.19 (Table 4) (Fig. 3).

\section{The utility of DW-MRI versus multiparametric MRI in the characterization of the studied 60 uterine cervix focal lesions}

The current study revealed that multiparametric MRI could detect all cervical focal lesions but with poor pathological characterization as most lesions (44/60) exhibit iso- to low signals at T1WI with intermediate-high signals at T2WI, and the remaining 16 lesions exhibit heterogeneous signals at both $\mathrm{T} 1$ and T2WI and postcontrast variable degree of enhancement achieving $72.37 \%$ sensitivity, $37.50 \%$ specificity, $63.33 \%$ accuracy, 76.19\% PPV, and 33.33\% NPV. When adding DWI with ADC value to MRI at high $b$ value $(b=800)$, it showed a higher diagnostic accuracy with good lesion pathological characterization that achieved $95.65 \%$ sensitivity, $71.43 \%$ specificity, 90\% accuracy, $91.67 \mathrm{PPV}$, and 83.33\% NPV (Table 5).

The presence of lymph node metastasis in the pelvic cavity was evaluated. Metastatic lymphadenopathy was defined as any enlarged lymph nodes larger than $10 \mathrm{~mm}$ in the short-axis diameter on T1-weighted images [10] (Fig. 4).

Table 2 Multiparametric MRI findings in the all studied uterine cervix focal lesions (number $=60$ )

\begin{tabular}{|c|c|c|c|c|}
\hline Lesions & No. & $\%$ & T1WI signals & T2WI signals \\
\hline Cervix Ca $(23,76.7 \%)$ & $\begin{array}{l}30 \\
16\end{array}$ & $\begin{array}{l}50 \\
26.7\end{array}$ & $\begin{array}{l}\text { Iso-low } \\
\text { Heterogeneous }\end{array}$ & $\begin{array}{l}\text { Moderate hyper-intense } \\
\text { Heterogeneous }\end{array}$ \\
\hline Cerxix polyp (4) & 8 & 13.3 & Iso-low & Moderate hyper-intense \\
\hline Ch. cervicitis with Nab. cyst & 4 & 6.5 & Iso-low & Mixed signals \\
\hline Cervix fibroid & 2 & $3.3 \%$ & Low & Mixed signals \\
\hline Total & \multicolumn{3}{|c|}{$\mathrm{No}=60$} & $100 \%$ \\
\hline
\end{tabular}


Table 3 DW-MRI and ADC values results of the 46 malignant lesions (group I)

\begin{tabular}{lllll}
\hline Lesion & No. & DWI $(b=800)$ & ADC map & ADC value \\
\hline Cerv. SC Ca & 34 & Intermediate-high & Low SI & $\begin{array}{l}0.89 .7-1.21 \\
\text { Mean }=0.1 \pm 0.09\end{array}$ \\
Cerv. End. Ca & \multirow{2}{*}{ High SI } & Low SI & $0.9-1.17$ \\
& & & Mean $=1 \pm 0.18$ \\
Cerv. Adeno. Ca & 4 & High SI & Low SI & $1-1.2$ \\
Total malig. lesions & \multirow{2}{*}{46} & Intermediate-high & Low SI & $0.86-1.1$ \\
& & & & Mean $=0.92 \pm 0.71$ \\
\hline
\end{tabular}

\section{Discussion}

The new 2018 FIGO system promotes the value of imaging modalities especially MRI imaging to increase the accuracy of tumor staging and guide treatment/monitoring. DWI increases the staging accuracy of MRI by permitting better evaluation of tumor size, extra uterine extension, and nodal infiltration, factors that affect treatment selection and planning [11-13].

In this study, the age of the included patients with suspected cervical cancers ranged from 39 to 63 years, $\mathrm{M}= \pm 44.6$ years. The abnormal vaginal bleeding was the main complaint in 42 patients while 18 patients complained of malodorous discharge and vaginal discomfort. This was in agreement with Sherif HA et al., who studied 20 female patients age $30-75$ years with a clinical picture of abnormal vaginal bleeding and vaginal discharge [14], and also with Rezvani $M$ et al. who stated that cancer cervix typically presents in younger women with an average age around 45 years, presented by abnormal vaginal bleeding, discomfort, and malodorous discharge as the first complaints [6].

We classified the studies 60 cases according to the histopathological results into 2 groups: 46 malignant lesions (group 1), 34 squamous cell carcinoma lesions, 8 adenocarcinomas, and 4 undifferentiated carcinoma and
14 benign lesions (group 2), 8 cervical polyps, 4 chronic cervicitis with nabothian cysts, and 2 fibroid lesions.

Our findings were in coincidence with that of Mahmoud SM et al., who found that the pathology of the included cases was sq. cell carcinoma in $72 \%$, adenocarcinoma in $12 \%$, sarcomatoid cervical carcinoma in $4 \%$, spindle cell tumor in $4 \%$, basaloid carcinoma in $4 \%$, and undifferentiated carcinoma in $4 \%[15]$, and with Dashottar S et al., who reported that out of the studied 35 cases, 33 (94.3\%) were sq. cell carcinoma, 1 (2.9\%) was papillary adenocarcinoma, and 1 (2.9\%) was small cell carcinoma [16]. However, Sherif HA et al. found that the histopathological diagnosis revealed adenocarcinoma in 4 cases $(20 \%)$, squamous cell carcinoma in 15 cases (75\%), and chronic cervicitis in 1 case (5\%) [14].

MR imaging represents the most valuable imaging modality for the detection of the primary tumor, nodal involvement, and local spread. It is also the best modality for showing recurrent disease and monitoring therapeutic response [17].

In the present work, the imaging findings revealed poor MRI signal characterization of pathologically proved different lesions as 27/30 cervical lesions showed iso- to hypointense signals on T1WI and moderate hyper-intense signals on T2WI with variable contrast

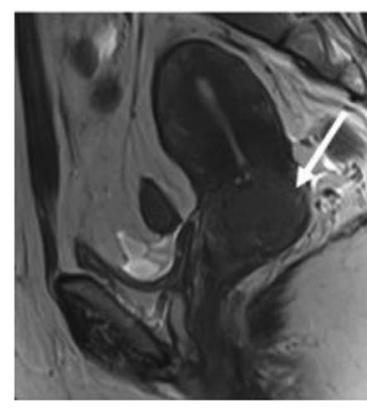

A

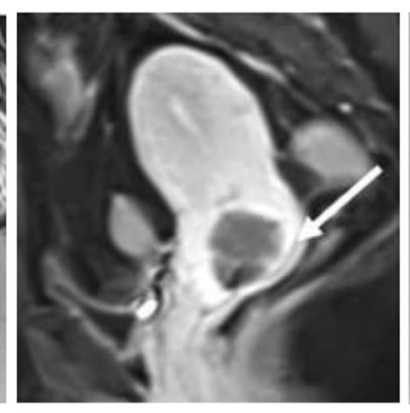

B

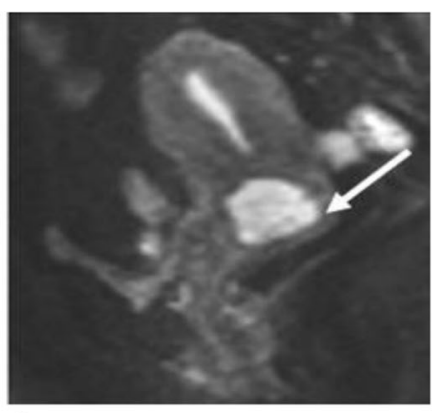

C

Fig. 1 A 42-year-old female patient presented with vaginal bleeding. MRI was done. a Sagittal T2WI revealed a relatively hyperintense signal focal lesion compared with the surrounding cervical stroma seen extending along the endocervix and endocervical canal. $\mathbf{b}$ Late post-contrast sagittal T1WI, the lesion showed non-homogenous enhancement at the late post-contrast study. c DWI, the lesion showed restricted diffusion with high b value (800) and corresponding low signal at ADC map. Diagnosis: cervical carcinoma 

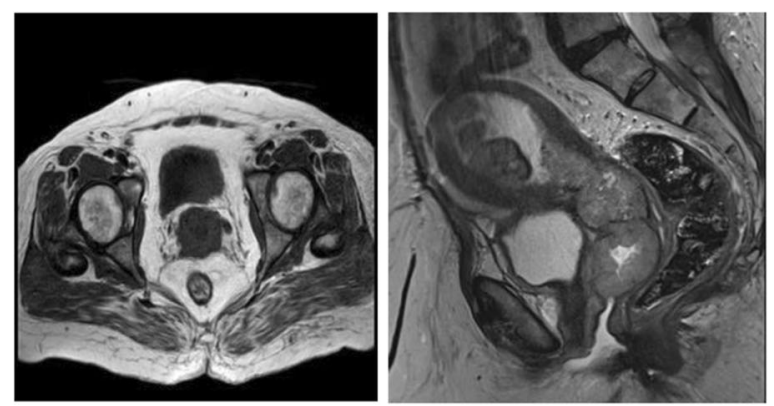

A

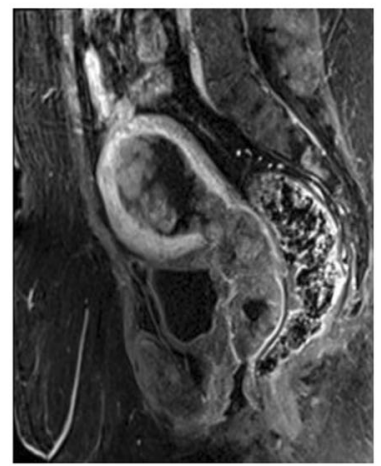

D
B

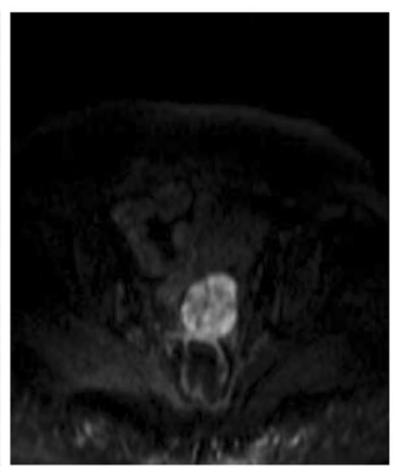

$\mathrm{E}$

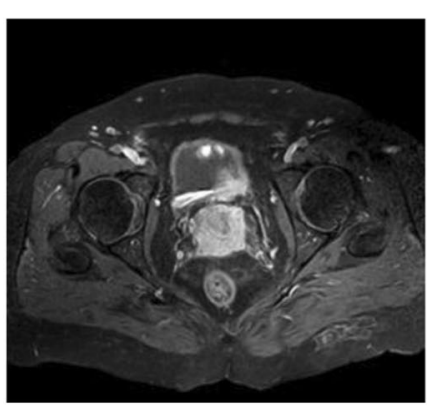

C

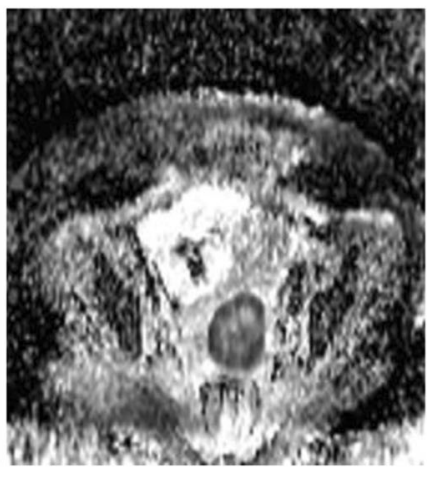

$\mathrm{F}$

Fig. 2 A 49-year-old female patient presented with vaginal bleeding. MRI pelvis was done. a, b Axial T1WI and sagittal T2WI revealed irregular polypoidal intermediate signal intensity lesion seen mainly harboring the cervical canal extending downward to the upper portion of the vagina and upward to the endometrial cavity with a subsequent irregular widening of the cervical canal and uterine cavity. c, $\mathbf{d}$ Axial and sagittal late post-contrast, the lesion showed non-homogenous irregular enhancement at the late post contrast sequence with invasion of the parametrium. e, f DWI, the lesion showed signal restriction with high $b$ value (800) with corresponding low signal at ADC map. Diagnosis: cervical carcinoma

enhancement regardless of benign or malignant etiology; however, 6 lesions (4 chronic cervicitis with nabothian cyst and 2 degenerated leiomyoma) showed iso- to low signal on T1WI and mixed/intermediate-high signals in T2WI.

Our findings were in concordance with Yoshikazu O who stated that some tumors or tumor-like lesions can show similar MR imaging findings, such as adenocarcinoma, adenoma malignum, and florid endocervical hyperplasia [18]. However, Mahmoud SA found that hypointense T1 signal was seen in 34/70 (48.5\%) cases, isointense signal in 28/70 (40\%) cases, hyperintense T1 signal in 6/70 (8.5\%) cases, and heterogeneous signal intensity in $2 / 70(2.8 \%)$. T2WIs showed a hyperintense signal in $62 / 70(88 \%)$ cases and intermediate signal in $2 / 70$
(2.8\%) cases and heterogeneous signal intensity in $6 / 70$ (8.5\%) [19]. Tamai KT et al. found that degenerated leiomyomas (7 lesions) showed low SI on T1-weighted images with areas of high SI on T2-weighted images [20]. Patel et al. reported that cervical tumors tend to give iso- to high signal compared to cervical stroma on T2WI [21].

The diffusion-weighted image (DWI) visualizes the local microstructural characteristics of water diffusion. High intensity on DWI with low apparent diffusion coefficients (ADC) is suggestive for malignant polyp with hypercellular nature, whereas benign polyps tend to show higher ADC value [22]. Our results revealed that all studied malignant cervical focal lesions $(n=46)$ showed positive diffusion restriction at high $b$ value $(b=$

Table 4 DWI and ADC map findings in 14 benign uterine cervix focal lesions (group II)

\begin{tabular}{lllll}
\hline Lesion & No. & DWI $(\mathrm{b} 800)$ & ADC map & ADC value \\
\hline Cerv. polyp & 8 & Low signals & High Sl at low $b$ value that fade out at high value & $1.37-1.64, M=1.4 \pm 0.31$ \\
Ch, cervicitis with Nab. cyst & 4 & Mixed signals & Mixed signals & $1.2-1.8, M=1.5 \pm 0.31$ \\
Deg, fibroid & 2 & Mixed signals & Mixed signals & 0.86 \\
Total benign lesions & 14 & Low-mixed signals & Mixed-high signals & $0.98-1.23$, mean $=1.18 \pm 0.1$ \\
\hline
\end{tabular}



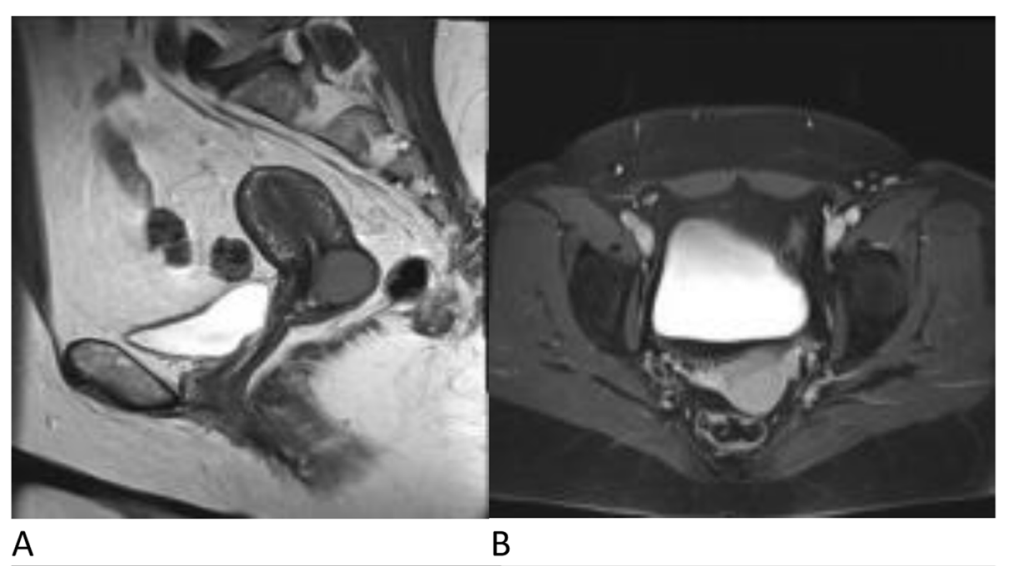

B

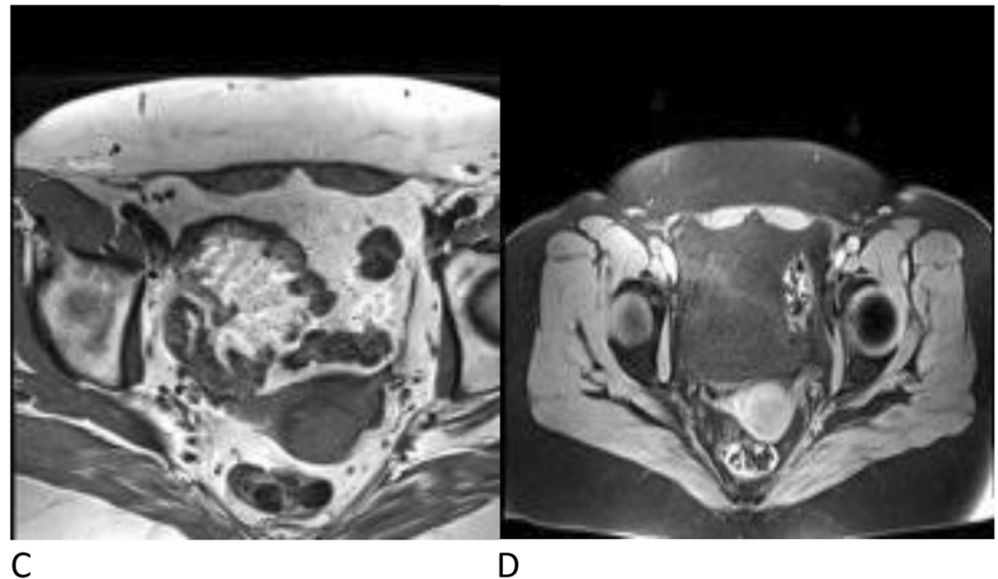

Fig. 3 A 42-year-old female patient presented with vaginal bleeding. MRI pelvis was done. a, b Sagittal and axial T2WI revealed a homogenously isointense abnormal signal intensity focal lesion with a well-circumscribed margin seen involving the posterior wall of the uterine cervix mildly encroaching upon the endocervical canal. c Axial T1WI, the lesion showed no areas of hemorrhage or degeneration. $\mathbf{d}$ Late post-contrast T1WI, the lesion showed homogenous contrast enhancement. Diagnosis: benign cervical lesion mostly cervical fibroid (myoma)

800) with relatively low ADC values that ranged from 0.86 to $1.15 \times 10^{-3} \mathrm{~mm}^{2} / \mathrm{s}, \mathrm{M}=0.92 \pm 0.71 \times 10^{-3} \mathrm{~mm}^{2} /$ s, while $8 / 14$ benign cervical lesions were diffusion negative in high $b$ value $(b=800)$ and 3 lesions $(4 \mathrm{ch}$. cervicitis with nabothian cyst and 2 degenerated fibroid) showed mixed diffusion changes. The ADC value of 10/ 14 benign focal lesions were relatively high that ranged from 1.57 to $2.4 \times 10^{-3} \mathrm{~mm}^{2} / \mathrm{s}\left(\mathrm{M}=1.7 \pm 0.31 \times 10^{-3}\right.$ $\mathrm{mm}^{2} / \mathrm{s}$ ); however, atypical lesions (chronic cervicitis with nabothian cyst and degenerated fibroid) showed low ADC values that ranged from 0.86 to $1.2 \times 10^{-3} \mathrm{~mm}^{2} / \mathrm{s}$ $(\mathrm{M}=0.1 \pm 0.19)$. This is may be attributed to the presence of necrosis and focal signal intensity changes as well as susceptibility artifacts. These result findings matched with many other studies which concluded the reported mean ADC values for both squamous cell carcinoma and adenocarcinoma cases to be less than 1 $\times 10^{-3} \mathrm{~mm}^{2} / \mathrm{s}$, averaging $0.88-0.91 \times 10^{-3} \mathrm{~mm}^{2} / \mathrm{s}, 0.8827$ $\times 10^{-3} \mathrm{~mm}^{2} / \mathrm{s}$, and $0.72 \pm 0.168 \times 10^{-3} \mathrm{~mm}^{2} / \mathrm{s}$, respectively [23-25]. Also, Mahmoud SA concluded that in the present study, the mean ADC value for malignant lesions was $0.82 \times 10^{-3} \pm 0.1 \mathrm{SD} \mathrm{mm} / \mathrm{s}$ [18], and Sherif $\mathrm{HA}$ et al. stated that the mean $\mathrm{ADC}$ value of cervical carcinoma in our study was $0.82 \times 10^{-3} \mathrm{~mm}^{2} / \mathrm{s}$ [14].

According to these study results, multiparametric MRI could detect all cervical focal lesions but with poor pathological characterization, achieving $72.37 \%$ sensitivity, $37.50 \%$ specificity, $63.33 \%$ accuracy, $76.19 \%$ PPV, and

Table 5 Validity of multiparametric MRI/DW-MRI in diagnosis of all studied endometrial lesions ( $n o=60$ )

\begin{tabular}{llllll}
\hline & Sensitivity (\%) & Specificity (\%) & Accuracy (\%) & PPV (\%) & PPV (\%) \\
\hline Multip. MRI & 72.37 & 37.5 & 63.33 & 76.19 & 33.33 \\
MRI + DWI & 95.65 & 71.43 & 90 & 91.67 & 83.33 \\
\hline
\end{tabular}




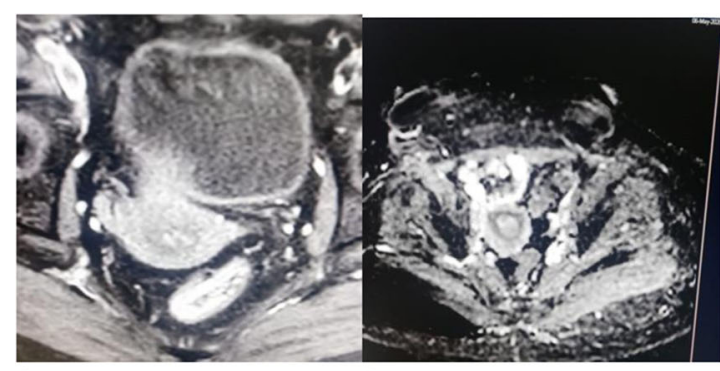

A

B

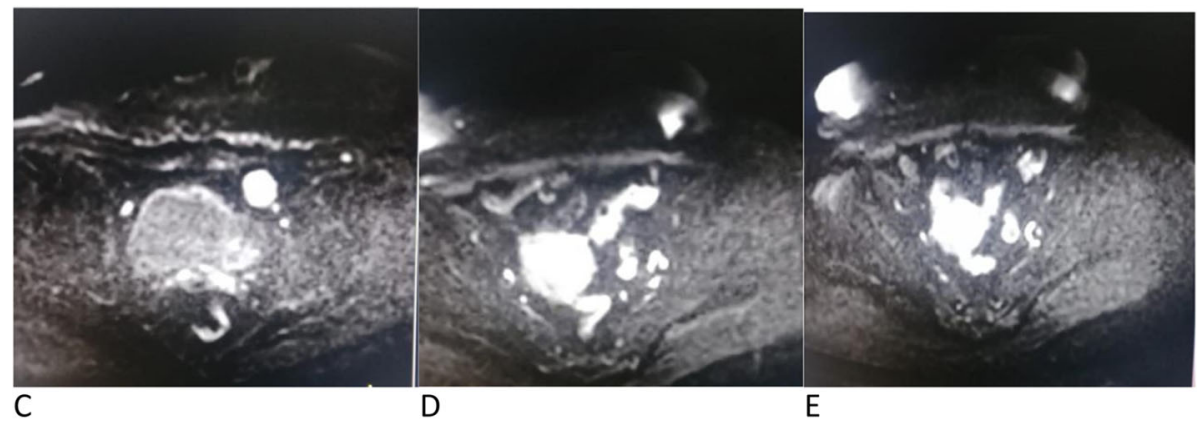

Fig. 4 A 50-year-old female patient post-operative status proved histopathologically as cervical carcinoma under treatment presented with vaginal bleeding. a Axial T2WI revealed an irregular hyperintense abnormal signal intensity lesion seen involving the operative bed as well as enlarged left external iliac LN. b Late post-contrast T1WI, the lesion showed irregular enhancement at the late post contrast study. c-e DWI, the lesion showed signal intensity restriction with high $b$ value (800) as well as hyperintense left external iliac LN with the corresponding low signal at ADC map. Diagnosis: recurrent cervical carcinoma

33.33\% NPV. When adding DWI with ADC value measurements at high $b$ value $(b=800)$ to MRI exam, it showed a higher diagnostic accuracy with good lesion pathological characterization that achieved $95.65 \%$ sensitivity, 71.43\% specificity, 90\% accuracy, $91.67 \mathrm{PPV}$, and 83.33\% NPV. Our results matched with Mahmoud SA et al. results which revealed that DWI-MRI showed sensitivity $100 \%$, specificity $50 \%$, accuracy $97 \%$, PPV $97 \%$, and NPV $100 \%$, and the low percentage of the specificity in this study was due to the low number of true negative patients [19], and Chen et al. found that the sensitivity and specificity of DW-MRI for tumor detection were $100 \%$ and $84.8 \%$, respectively. DWI showed $100 \%$ sensitivity, a positive predictive value [26]. Mahmoud SM reported that DW-MRI revealed sensitivity, specificity, PPV, and accuracy of $100 \%, 50 \%, 97 \%$, and $97 \%$ respectively [18]. Also, Exner M. et al. concluded that the use of DWI led to an increase in sensitivity of infiltrated adjacent tissue (from 86 to 90\%) and detection of lymph node metastases (from 47 to $67 \%$ ) [27].

\section{Limitations}

The main limitation of this study was the limited sample size. Further studies using a cohort study of patients with long-term follow-up are needed to establish the accuracy of this method for early detection of cervical uterine cancer.

\section{Conclusion}

Adding DWI with ADC values measurements at high $b$ value to the multiparametric MRI examination of the female pelvis increases the sensitivity, specificity, and diagnostic accuracy for characterization and early diagnosis of cervical malignant focal lesions and appropriate planning management options.

DWI may replace the contrast study especially for patients not candidates for contrast injection. It can be used in conjunction with ADC values as a standard routine protocol after application in a large number of patients aiming to decrease the diagnostic pitfalls and get higher diagnostic performance including distant metastatic infiltration.

\section{Abbreviations}

ADC: Apparent diffusion coefficient; DWI: Diffusion-weighted images; FOV: Field of view; FSE: Fast spin echo; MRI: Magnetic resonance imaging; NPV: Negative predictive value; PPV: Positive predictive value; TE: Time of echo; TR: Time of repetition; ROI: Region of interest; SI: Signal intensity

\section{Acknowledgements}

Not applicable

\section{Authors' contributions}

MAE collected and analyzed the data and sharing in the results and discussion. NMO completed the results, discussion, and conclusion. All authors read and approved the final manuscript.

\section{Funding}

No available funding for this research. 


\section{Availability of data and materials}

All data generated or analyzed during this study are included in this published article [and its supplementary information files].

\section{Ethics approval and consent to participate}

Our ethics committee guidelines are applied to all patients according to the Egyptian Network of Research Ethics Committee (ENREC).

Committee reference number: not available.

All patients had written informed consent.

\section{Consent for publication}

Every patient had written informed consent for publication of his medial data.

\section{Competing interests}

The authors declare that they have no competing interests.

Received: 21 October 2020 Accepted: 18 January 2021

Published online: 02 February 2021

\section{References}

1. Reinhold C, Gallix BP, Ascher SM (1997) Uterus and cervix. In: Semelka RC, Ascher SM, Reinhold C (eds) MRI of the abdomen and pelvis: a text atlas. Wiley-Liss, New York, pp 585-660

2. McCarthy $S$, Hricak $H$. The uterus and vagina. In:Higgins $C B$, Hricak H, Helms CA, eds. Magnetic resonance imaging of the body. 3rd ed. New York, NY: Lippincott-Raven, 1997; 761-814.

3. Bazot M, Darai E, Nassar-Slaba J et al (2008) Value of magnetic resonance imaging for the diagnosis of female pelvis tumors: a review. J Comput Assist Tomogr 32(5):712-723

4. Sohaib SA, Mills TD, Sahdev A et al (2005) The role of magnetic resonance imaging and ultrasound in patients with adnexal masses. Clin Radiol 60(3): 340-348

5. Solomon D, Breen N, McNeel T (2007) Cervical cancer screening rates in the United States and the potential impact of implementation of screening guidelines. CA Cancer J Clin 57(2):105-111

6. Rezvani M, Shaaban A (2009) Imaging of cervical pathology. Clin Obstet Gynecol 52(1):94-111

7. Whittaker C, Coady A, Culver L et al (2009) Diffusion weighted MR Imaging of female pelvic tumors: a pictorial review. Radio Graphics 29(3):759-778

8. Kuang F, Ren J, Huan Yet al. (2012) Apparent diffusion coefficients of normal uterus in premenopausal women with 3.0-T magnetic resonance imaging. J Comput Assist Tomogr 36(1):54-59

9. Namimoto T, Awai K, Nakaura T et al (2009) Role of diffusion-weighted imaging in the diagnosis of gynecological diseases. Eur Radiol 19(3):745760

10. Perucho JAU, Chiu KWH et al (2020) Diffusion-weighted magnetic resonance imaging of primary cervical cancer in the detection of subcentimetre metastatic lymph nodes. Cancer Imaging 20:27

11. Sala E, Rockall A, Rangarajan D et al (2010) The role of dynamic contrastenhanced and diffusion weighted magnetic resonance imaging in the female pelvis. Eur J Radiol 76(3):367-385

12. McVeigh PZ, Syed AM, Milosevic M et al (2008) Diffusion-weighted MRI in cervical cancer. Eur Radiol 18(5):1058-1064

13. Mourad MA-F, Mousa EM (2017) Comparing T2 weighted images/diffusion weighted imaging and T2 weighted images/dynamic contrast enhanced MRI for endometrial carcinoma myometrial invasion. Egypt J Radiol Nuclear Med 48:323-327

14. Sherif H Abo Gamra, Mohamed G Abdel Mutaleb, Haydar M Sinjawi. (2018) Role of diffusion weighted MRI in diagnosis of cervical cancer. Egypt $\rfloor$ Hospital Med 71 (1):2411-2421.

15. Mahmoud SM, Raafat M (2017) Is there an added role for diffusion weighted imaging in the staging of cervical carcinoma? Egypt J Radiol Nucl Med 48:1131-1139

16. Dashottar S, Preeth TP, Lohia N (2019) Role of apparent diffusion coefficient as a biomarker in the evaluation of cervical cancer. Indian J Radiol Imaging 29(1):25-32

17. Dappa E, Elger T, Hasenburg A et al (2017) The value of advanced MRI techniques in the assessment of cervical cancer: a review. Insights Imaging $8(5): 471-481$
18. Yoshikazu O, Yumiko OT, Masato $\mathrm{N}$ et al (2003) MR Imaging of the uterine cervix: imaging-pathologic correlation. RadioGraphics 23:425-445

19. Abd elsalam SM, Mokhtar O, Lamia Adel L et al. (2020) Impact of diffusion weighted magnetic resonance imaging in diagnosis of cervical cancer. Egypt J Radiol Nucl Med 51:23

20. Tamai K, Koyama T, Saga T et al (2008) The utility of diffusion-weighted MR imaging for differentiating uterine sarcomas from benign leiomyomas. Eur Radiol 18(4):723-730

21. Patel S, Liyanage S, Sahdev A et al (2010) Imaging of endometrial and cervical cancer. Insights Imaging 1(5-6):309-328

22. Takeuchi M, Matsuzaki K, Harada M et al (2011) Differential diagnosis of uterine polyps: MR manifestations of various epithelial and mesenchymal tumors and tumor like lesions, pitfalls and problem-solving MR techniques. Poster No.: C-1908. Congress: ECR 2011. ESOR., EPOS. https://doi.org/10. 1594/ecr2011/C-1908)

23. Hasan DI, Enaba MM, Abd El-Rahman HM, El-Shazely S (2015) Apparent diffusion coefficient value in evaluating types, stages and histologic grading of cancer cervix. Egypt J Radiol Nucl Med 46:781-789

24. Kala P, Bhargavi V, Avantsa R, Narayan G (2017) Role of diffusion weightedmagnetic resonance imaging in cervical cancer for prediction and monitoring of chemo-radiotherapy. Universal journal of medical science. Vol. 5(1):13-21

25. Karunya RJ, Tharani P, John S, Kumar RM, Das S (2017) Role of functional magnetic resonance imaging derived parameters as imaging biomarkers and correlation with clinicopathological features in carcinoma of uterine cervix. J Clin Diagn Res 8:XC06-XC11

26. Chen J, Zhang Y, Liang B et al (2010) The utility of diffusion-weighted MR imaging in cervical cancer. Eur J Radiol 74:e101-e106

27. Exner M, Kühn A, Stumpp P et al (2015) Value of diffusion-weighted MRI in diagnosis of uterine cervical cancer: a prospective study evaluating the benefits of DWI compared to conventional MR sequences in a 3T environment. Acta Radiologica 57(7):869-877

\section{Publisher's Note}

Springer Nature remains neutral with regard to jurisdictional claims in published maps and institutional affiliations.

\section{Submit your manuscript to a SpringerOpen ${ }^{\circ}$ journal and benefit from:}

- Convenient online submission

- Rigorous peer review

- Open access: articles freely available online

- High visibility within the field

Retaining the copyright to your article

Submit your next manuscript at $>$ springeropen.com 